\title{
Salvage therapy for colonic mechanical obstruction with introducer-type colostomy and colopexy
}

Georgios Mavrogenis, MD, Ioannis Tsevgas, MD, George Theodoropoulos, MD, PhD,

Dimitrios Zachariadis, MD

Percutaneous endoscopic colostomy (PEC) has been established as an alternative method of intestinal decompression and irrigation for functional large bowel obstruction and relapsing sigmoid volvulus. However, postoperative fecal peritonitis secondary to stool leakage has been reported in $5 \%$ to $12 \%$ of cases. ${ }^{1}$ Prior fixation of the colon to the anterior abdominal wall with the help of a double-needle suturing device or $\mathrm{T}$ fasteners could eventually reduce that risk. ${ }^{2-4}$

This video (Video 1, available online at www.VideoGIE. org) presents the case of a 70-year-old man with a 2-month history of diarrhea and painful abdominal distention not responding to medical treatment. He had previously undergone anterior resection for rectal cancer,

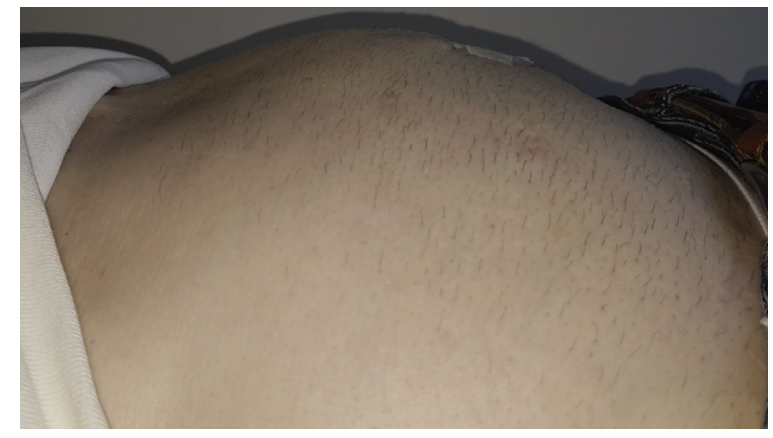

Figure 1. Distended abdomen as shown by clinical examination.

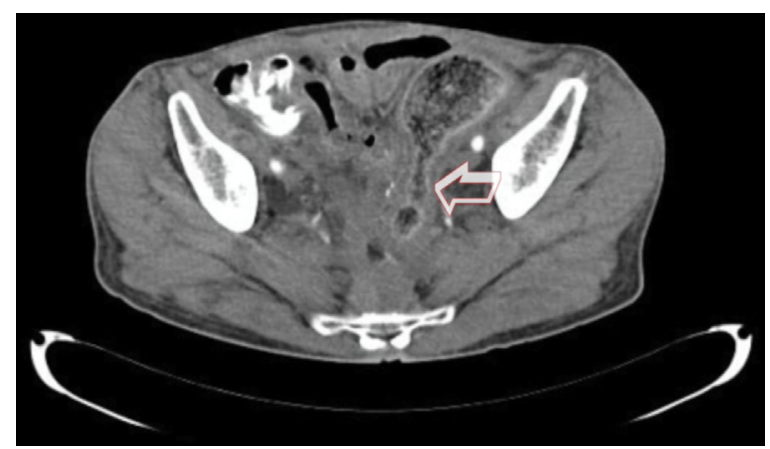

Figure 2. CT image showing a $7-\mathrm{cm}$ long stricture of the neorectum. radiotherapy for bladder cancer, and resection of postradiation small-intestinal strictures. Clinical examination revealed a cachectic patient with significant distention and high-pitched bowel sounds (Fig. 1). CT disclosed a 7-cm-long stricture of the neorectum with upstream dilation of the colon up to $10 \mathrm{~cm}$ (Figs. 2 and 3). Colonoscopy with a

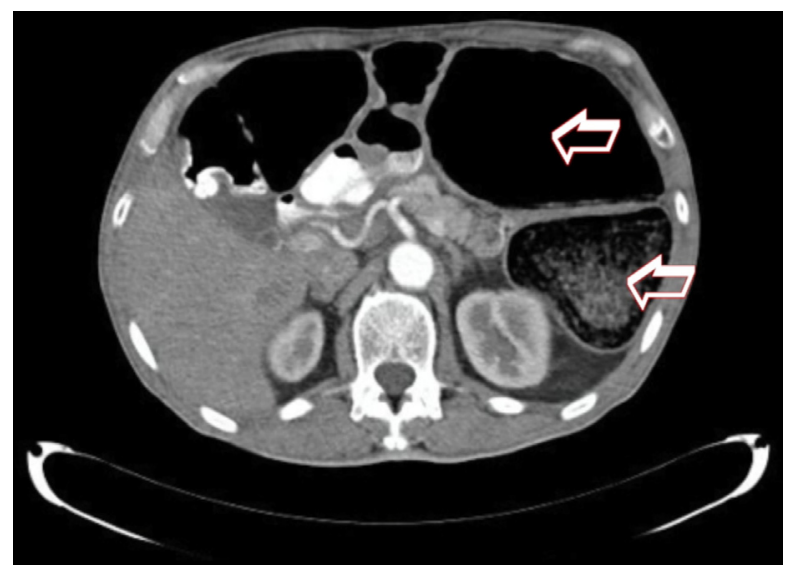

Figure 3. CT image showing dilation of the transverse and left side of the colon up to $10 \mathrm{~cm}$.

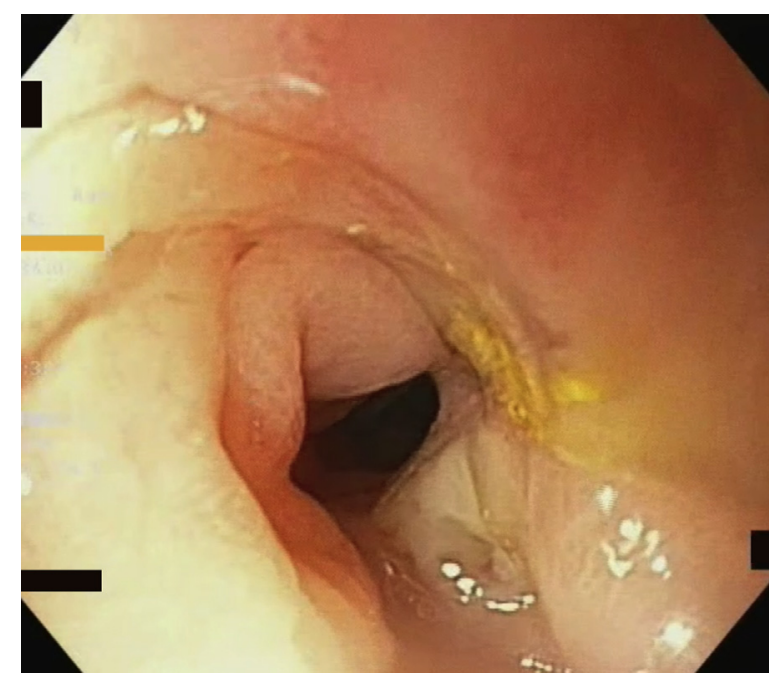

Figure 4. Endoscopic image of the stricture. 


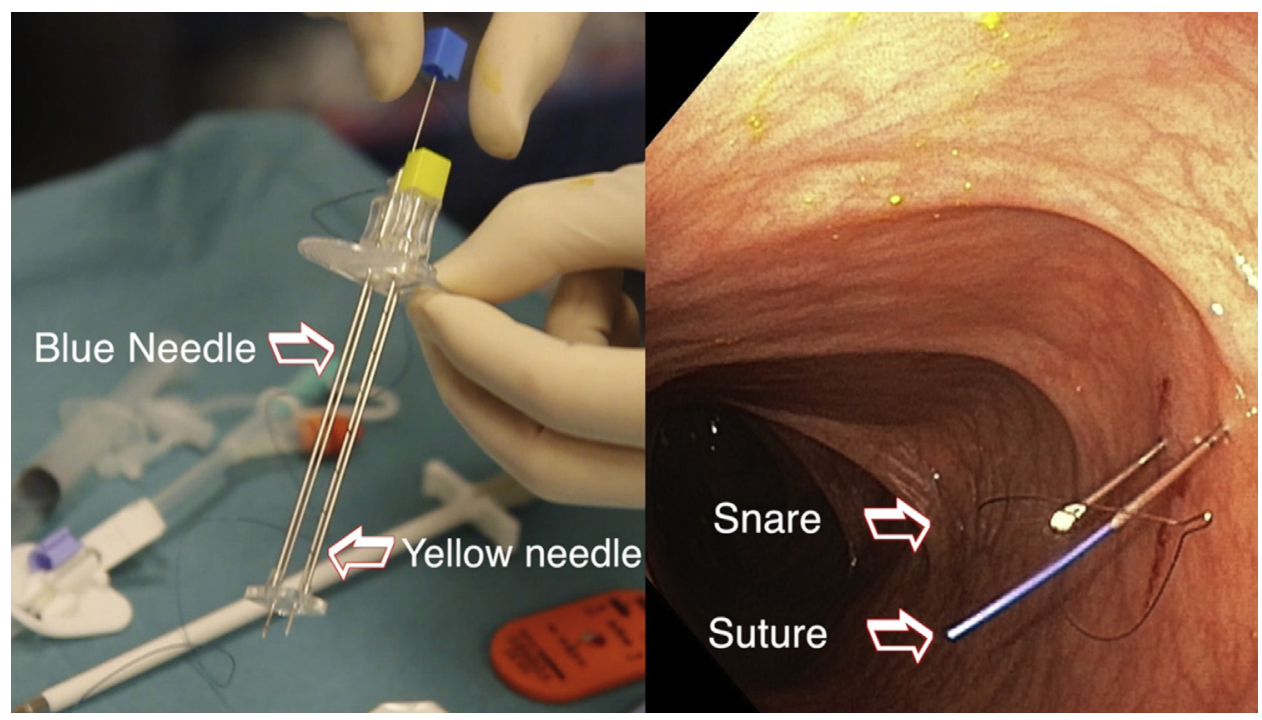

Figure 5. Double-needle device. The blue needle contains a snare. The needle with the yellow tip contains the suture.

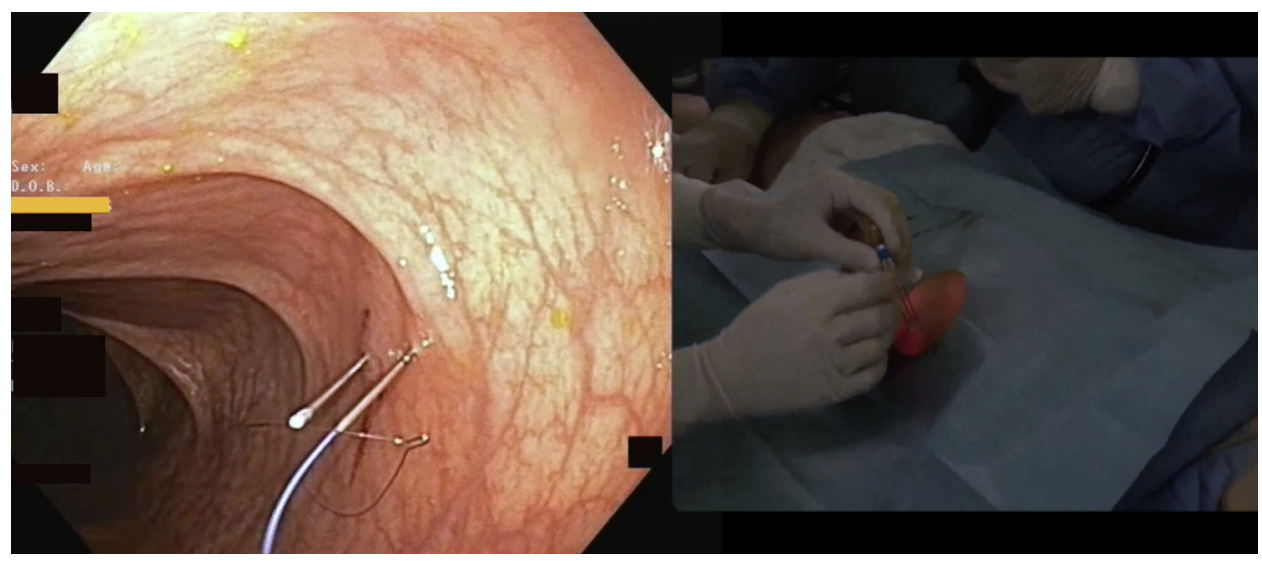

Figure 6. Colopexy with the double-needle system.

slim endoscope revealed a long stricture involving the neorectum and sigmoid colon that had not been present before radiotherapy (Fig. 4). Endoscopic treatment with stenting was avoided because of the benign nature of the stricture, its long length, the short distance from the anus, and the potential for side effects such as tenesmus and migration. Furthermore, we estimated that pneumatic dilation of such a long postradiation stricture carried a significant risk of perforation. The patient was deemed unfit to undergo surgery. Therefore, an introducer-type colostomy was suggested, combined with colopexy, with use of a dedicated suturing device (Fig. 5, Video 1) that has been designed for percutaneous endoscopic gastropexy (Freka Pexact Gastrostomy system, Fresenius Kabi, Runcorn, Cheshire, United Kingdom). ${ }^{5}$

Prophylactic antibiotics were administrated before the procedure. The left side of the colon was distended with $\mathrm{CO}_{2}$, and the puncture site was located by means of light transillumination and finger indentation, proximally to the stenotic segment. Local anesthesia was administered, and the colon was punctured with the injection needle to confirm good positioning. Then, we performed colopexy with the help of the introducer needle, which was inserted in the lumen of the colon (Figs. 6 and 7). A small incision was made between the sutures, and a trocar with a peel-away sheath was introduced through the abdominal wall into the colon (Fig. 8). The trocar was removed, a 15F PEG tube was progressed through the sheath, and the balloon was inflated with $5 \mathrm{~mL}$ of sterile water (Fig. 9). The peel-away sheath was removed, and the retaining plate was placed.

The patient recovered uneventfully and was discharged after 24 hours. Antibiotics were continued for 5 more days, and the sutures were removed after 10 days. We advised the patient to flush the tube with at least $50 \mathrm{~mL}$ of water 3 times per day to avoid obstruction of the tube by stools and to open the valve of the tube as needed to exsufflate the distended abdomen. When necessary, he could flush the tube with larger quantities of water to facilitate defecation. 


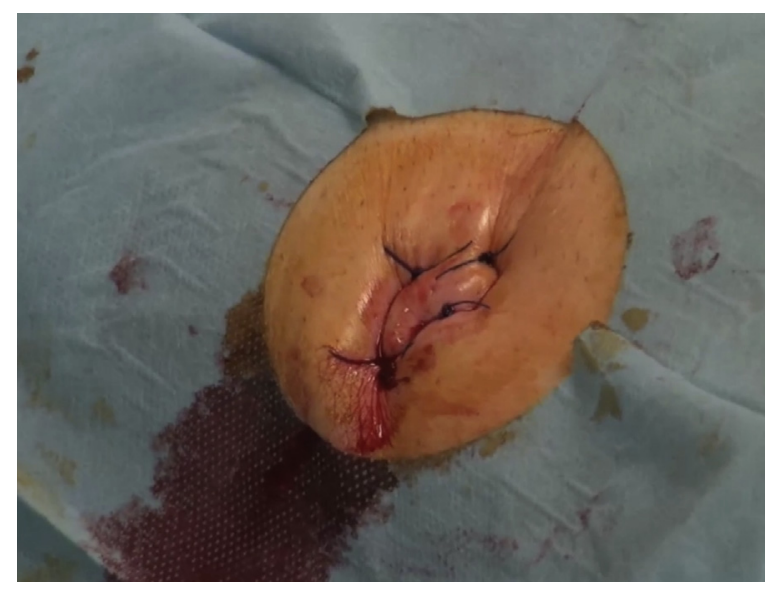

Figure 7. Placement of a total of 4 sutures.

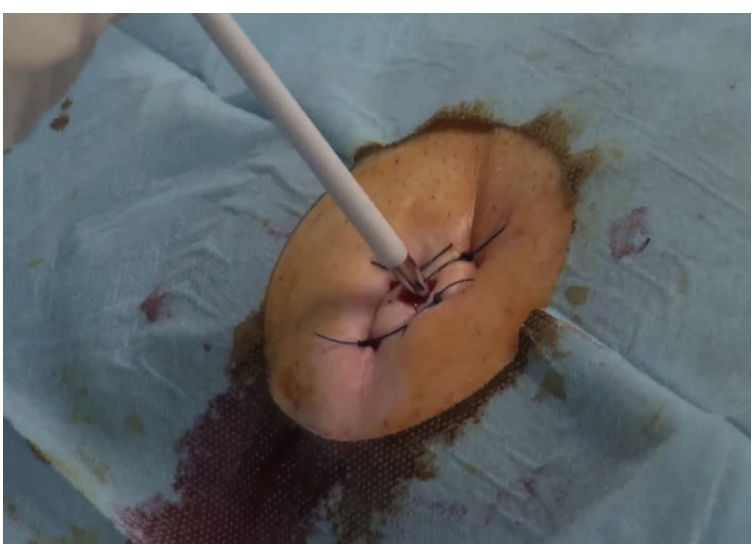

Figure 8. Advancement of a trocar with a peel-away sheath between the sutures.

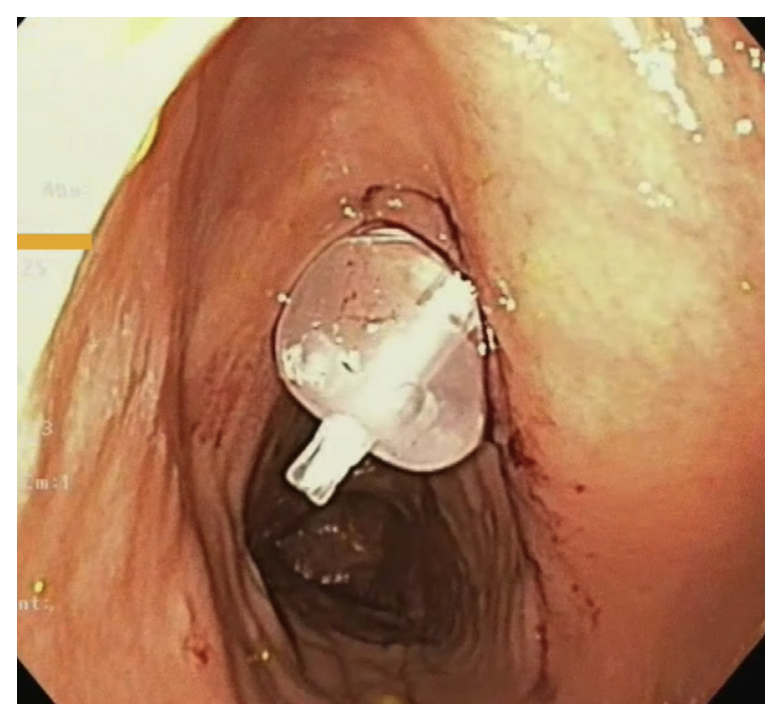

Figure 9. Endoscopic image of the $15 \mathrm{~F}$ tube.

After 2 months of regular use of the tube for both decompression and irrigation, the symptoms progressively improved. The patient was readmitted for replacement of the balloon catheter with a standard pull-through gastrostomy tube. However, the patient refused further endoscopic treatment, despite the risk of recurrence. The colostomy tube was removed, and the fistulous tract was closed 2 days later. At a 6-month follow-up visit, the patient had mild abdominal distention, and subsequent episodes of obstruction have been managed successfully with the combination of pancreatic enzyme supplements and osmotic laxatives.

In conclusion, PEC with the introducer method, combined with colopexy, may be used as salvage therapy for colonic decompression and irrigation in selected cases of mechanical obstruction. Fixation of the colon to the abdominal wall may decrease the risk of postoperative peritonitis, secondary to stool leakage or to tube dislocation.

\section{DISCLOSURE}

The production of this video was funded by the Greek Society of Gastroenterology. All autbors disclosed no financial relationships relevant to this publication.

\section{ACKNOWLEDGMENT}

The authors thank Mr Fragkos Georgios for technical support.

Abbreviation: PEC, Percutaneous endoscopic colostomy.

\section{REFERENCES}

1. Frank L, Moran A, Beaton C. Use of percutaneous endoscopic colostomy (PEC) to treat sigmoid volvulus: a systematic review. Endosc Int Open 2016;4:e737-41.

2. Molina-Infante J, Mateos-Rodriguez JM, Vinagre-Rodriguez G, et al. Endoscopic-assisted colopexy and push percutaneous colostomy in the transverse colon for refractory chronic intestinal pseudoobstruction. Surg Laparosc Endosc Percutan Tech 2011;21:e322-5.

3. López-Serrano A, Amurrio CA, Hervás J, et al. Endoscopic treatment of recurrent sigmoid volvulus with colopexy assisted by T-fasteners and colostomy. Endoscopy 2016;48:e236-7.

4. Moriwaki Y, Otani J, Okuda J, et al. Safe percutaneous endoscopic colostomy for severe constipation with use of the introducer method. Endoscopy 2015;47:e358-60.

5. Mavrogenis $G$, Kisoka $P$, Warzée $P$, et al. Video demonstration of the introducer-type percutaneous gastrostomy system. Gastrointest Endosc 2014;79:204.

Mediterraneo Hospital, Athens, Greece.

Copyright (c) 2017 The Authors. Published by Elsevier, Inc. on behalf of the American Society for Gastrointestinal Endoscopy. This is an open access article under the CC BY-NC-ND license (http://creativecommons.org/ licenses/by-nc-nd/4.0/).

Presented at the World Cup of Endoscopy at Digestive Disease Week, May 6-9, 2017, Chicago, Illinois.

http://dx.doi.org/10.1016/j.vgie.2017.01.012 